J. Math. Phys., Vol 38, No. 3, March 1997

\title{
The ultrarelativistic Reissner-Nordstrøm field in the Colombeau algebra
}

\author{
R. Steinbauer \\ Institute for Theoretical Physics, University of Vienna \\ Boltzmanng. 5, A-1090 Wien/Vienna, Austria \\ E-mail: stein@doppler.thp.univie.ac.at
}

(Received 9 May 1996; accepted for publication 12 November 1996)

The electromagnetic field of the ultrarelativistic Reissner-Nordstrøm solution shows the physically highly unsatisfactory property of a vanishing field tensor but a nonzero, i.e., $\delta$-like, energy density. The aim of this work is to analyze this situation from a mathematical point of view, using the framework of Colombeau's theory of nonlinear generalized functions. It is shown that the physically unsatisfactory situation is mathematically perfectly defined and that one cannot avoid such situations when dealing with distributional valued field tensors. PACS numbers: $04.40 . \mathrm{Nr}$, 02.30.Sa, 04.90+e, 02.90.+p

\section{INTRODUCTION}

Recently, there has been some interest in gravitational shock wave geometries produced by ultrarelativistic particles 13 In field and string theoryone is interested especially in high energy scattering processes in these geometries. 60

In 1971 Aichelburg and Sexld derived the ultrarelativistic limit of the Schwarzschild metric, which turns out to be a pp-wave with a distributional, i. e., $\delta$-like profile fupction. This result has been reproduced by several authors using various methods, 3 but all of them invoking distributional techniques. Loustó and Sánchera derived the ultrarelativistic limit of the Reissner-Nordstrøm solution. To gain a distributionally well defined limit of the metric they had to rescale the charge by $e^{2}=p_{e}^{2}\left(1-v^{2}\right)^{1 / 2}$ which also forces the limit of the electromagentic field to vanish (even in the space of distributions). However the limit of the energy stress tensor of the eletromagnetic field is nonvanishing, thus leaving us with the physically highly unsatisfactory situation of a vanishing field producing a $\delta$-like energy density.

The aim of this work is to discuss this situation from a more mathematical point of view. Computing the electromagnetic stress tensor from the field tensor is a nonlinear operation and thus not defined within the framework of classical distribution theory. Thus, strictly speaking, if one is dealing with distributional-valued feld tensors one has to go beyond distribution theory. In the 1980s J.F. Colombeaul 10 developed a theory of generalized functions providing the possibility of a product of distributions. He constructed differential algebras $\mathcal{G}$ containing the space of smooth functions as a subalgebra and the space of distributions as a subspace. Thus Colombeau's theory provides us with a natural framework for discussing situations like the above-mentioned one from a more abstract viewpoint 11.22 Moreover most recently first applications of this very framework to problems of classical field theories 13 and general relativity 14 have appeared. 
This work is organized in the following way. In Sec. II we briefly recall the basics of Colombeau's nonlinear theory of generalized functions. To get some working knowledge, in Sec. III we take a look at the ultrarelativistic limit of the Coulomb field in flat space, and finally in Sec. IV we discuss in some detail the electromagnetic field of the ultrarelativistic Reissner-Nordstrøm solution and its energy stress tensor.

\section{BASICS OF COLOMBEAU THEORY}

Let $\Omega$ be an open subset of $\mathbb{R}^{n}$ or a smooth manifold. We denote by $\mathcal{D}(\Omega)$ the space of test functions on $\Omega$, i. e., the space of smooth functions with compact support in $\Omega$, and by $\mathcal{D}^{\prime}(\Omega)$ the space of distributions on $\Omega$. Finally the action of a distribution $w$ on a test fuction $\varphi$ we denote by $\langle w, \varphi\rangle$.

In $\mathcal{D}^{\prime}$ no meaningful product can be defined. Moreover, as L. Schwartz showed in 1954 , there even exists no associative and commutative differential algebra containing the space of continuous functions as a subalgebfathat allows a linear embedding of the space of distributions in it. J.F. Colombear 10 introduced differential algebras $\mathcal{G}$ containing the space of distributions as a subspace, and the space of smooth functions as a faithful subalgebra, thus providing a natural framework for studying nonlinear operations on singular data, i. e., distributions.

In this paper we consider the "special variant" of Colombeau's algebra, whose definition we briefly recall. Set

$$
\begin{gathered}
\mathcal{E}_{\mathcal{M}}(\Omega)=\left\{\left(u_{\epsilon}\right)_{\epsilon} \in\left(C^{\infty}(\Omega)\right)^{(0,1)}: \forall K \subset \subset \Omega \forall \alpha \in \mathbb{N}_{0}^{n} \exists N>0:\right. \\
\left.\sup _{x \in K}\left|\partial^{\alpha} u_{\epsilon}(x)\right|=O\left(\epsilon^{-N}\right) \quad(\epsilon \rightarrow 0)\right\}, \\
\mathcal{N}(\Omega)=\left\{\left(u_{\epsilon}\right)_{\epsilon} \in\left(C^{\infty}(\Omega)\right)^{(0,1)}: \forall K \subset \subset \Omega \forall \alpha \in \mathbb{N}_{0}^{n}, \forall M>0:\right. \\
\left.\sup _{x \in K}\left|\partial^{\alpha} u_{\epsilon}(x)\right|=O\left(\epsilon^{M}\right) \quad(\epsilon \rightarrow 0)\right\} .
\end{gathered}
$$

$\mathcal{E}_{\mathcal{M}}(\Omega)$ is a differential algebra with pointwise operations and $\mathcal{N}(\Omega)$ is an ideal in it. We define the algebra of generalized functions, or Colombeau algebra, by

$$
\mathcal{G}(\Omega):=\mathcal{E}_{\mathcal{M}}(\Omega) / \mathcal{N}(\Omega)
$$

and denote its elements by

$$
u=\left(u_{\epsilon}\right)_{\epsilon}+\mathcal{N}(\Omega) .
$$

Distributions with compact support on $\mathbb{R}^{n}$ can now be embedded into $\mathcal{G}\left(\mathbb{R}^{n}\right)$ by convolution with a mollifier $\rho_{\epsilon}$, defined as follows; let $\rho \in \mathcal{S}\left(\mathbb{R}^{n}\right)$ (Schwartz's space) with the properties $\int \rho(x) d x=1$ and $\int x^{\alpha} \rho(x) d x=0 \quad \forall \alpha \in \mathbb{N}^{n},|\alpha| \geq 1$, then we set $\rho_{\epsilon}(x):=\left(1 / \epsilon^{n}\right) \rho(x / \epsilon)$. So we have the map $\iota_{0}(\omega)=\left(\omega * \rho_{\epsilon}\right)_{\epsilon}+\mathcal{N}\left(\mathbb{R}^{n}\right)$.

This embedding can be "lifted" to an embedding $\iota: \mathcal{D}^{\prime}(\Omega) \hookrightarrow \mathcal{G}(\Omega)$ by means of sheaf theory while smooth functions are embedded as constant sequences, i. e. $\sigma(f)=(f)_{\epsilon}$.

Next we briefly recall the concept of association in the Colombeau algebra, of which we make extensive use in the physical calculations of Secs. III and IV. One particular property of Colombeau's algebra is the so-called "coupled-calculus," namely equality in the algebra of generalized functions and equality on distribution level. The latter is defined as follows: a generalized function $u$ is called associated to 0 $(u \approx 0)$ if for one (and hence any) representative $\left(u_{\epsilon}\right)_{\epsilon}$ we have

$$
\lim _{\epsilon \rightarrow 0} \int u_{\epsilon}(x) \varphi(x) d x=0, \quad \forall \varphi \in \mathcal{D}(\Omega) .
$$


Association defines an equivalence relation in the Colombeau algebra that clearly is coarser than equality and is compatible with differentiation, i. e., $u \approx v \Rightarrow \partial^{\alpha} u \approx$ $\partial^{\alpha} v$ but is not compatible with the algebra multiplication, i. e., $u \approx v \nRightarrow w u \approx w v$.

If a generalized function $u$ is associated to $\iota(w)$, where $w$ is a distribution, then one says that $w$ is the distributional shadow or the associated distribution of $u$. Not all Colombeau functions have a shadow-for example $\delta^{2}$, i.e., $\iota(\delta)^{2}$-but if a generalized function has a shadow the latter is unique. Different generalized functions may have the same shadow, providing us with the possibility to model singular situations in various ways 10

\section{THE ULTRARELATIVISTIC LIMIT OF THE COULOMB FIELD}

In Cartesian coordinates the Coulomb field is given by the Maxwell field tensor components $F^{0 \alpha}=-\left(e x^{\alpha}\right) / r^{3}$ where $r^{2}=x^{2}+y^{2}+z^{2}$ and $\alpha=1,2,3$, and the other components vanishing. We apply a Lorentz boost with velocity $v$ in the $x$ direction and, in order to simplify computations, we additionally transform to a frame associated with null coordinates; in particular

$$
\begin{aligned}
t & \mapsto \bar{t}=\gamma(t+v x) \mapsto u=\bar{x}-\bar{t}, \\
x & \mapsto \bar{x}=\gamma(x+v t) \mapsto w=\bar{x}+\bar{t},
\end{aligned}
$$

where $\gamma:=\left(1-v^{2}\right)^{-1 / 2}$. Thus the boosted field tensor takes the form

$F^{i k}=\frac{e\left(1-v^{2}\right)}{\left[(\bar{x}-v \bar{t})^{2}+\left(1-v^{2}\right) \rho^{2}\right]^{3 / 2}}\left(\begin{array}{cccc}0 & 2(\bar{x}-v \bar{t}) & (1-v) y & (1-v) z \\ -2(\bar{x}-v \bar{t}) & 0 & -(1+v) y & -(1+v) z \\ -(1-v) y & (1+v) y & 0 & 0 \\ -(1-v) z & (1+v) z & 0 & 0\end{array}\right)$,

where $\rho^{2}=y^{2}+z^{2}$.

We could now view the components of the field tensor as sequences of locally integrable functions parametrized by the boost velocity $v$, and calculate their limits in the space of distributions as $v \rightarrow 1$. However, it is well known that in this limit some components of the field tensor tend to $\delta(u)$ and hence the energy momentum tensor $T_{i k}=F_{i l} F_{k}^{l}-1 / 4 F_{m l} F^{l m}$ of the ultrarelativistic field cannot be calculated directly due to the lack of a multiplication in the space of distributions. Alternatively one could think of first computing the energy momentum tensor of the boosted field and then taking the limit. However, in this approach some components of the momentum tensor diverge in the space of distributions, thus making it again impossible to compute the energy momentum tensor of the ultrarelativistic Coulomb field within the framework of classical distribution theory.

To overcome these difficulties we embed the components $\left(F^{i k}{ }_{v}\right)_{v}$ of the field tensor into the Colombeau algebra. The physically most natural way to do so is to view a whole sequence (for example $\left(F^{01} v\right)_{v}$ ) as one Colombeau function. Generalized functions in the sense of Colombeau are classes of sequences of smooth functions, but our sequences are not even defined on the set $\mathcal{A}=\mathbb{R}^{4} \backslash\{x=v t(0<$ $v<1) \wedge \rho=0\}$, where from now on we neglect the bars over $x$ and $t$ to simplify the notation. So in order to get an "embedding" of $\left(F^{i k}\right)_{v}$ into Colombeau's algebra without cutting the whole set $\mathcal{A}$ from the domain we employ the following construction that only forces us to restrict the domain to $\Omega:=\mathbb{R}^{4} \backslash\{x=t \wedge \rho=$ $0\}$, which is physically reasonable since we precisely cut out the world line of the ultrarelativistic particle. Let $\mathcal{X}_{v} \in C^{\infty}(\mathbb{R})(0<v<1)$ be such that

$$
\begin{array}{ll}
\mathcal{X}_{v}(\xi) \equiv 1, & |\xi| \geq 4(1-v)^{2}, \\
\mathcal{X}_{v}(\xi) \equiv 0, & |\xi| \leq(1-v)^{2} .
\end{array}
$$


Using the abbreviation $a:=(x+v t)^{2}+\rho^{2}$ we define

$$
\tilde{F}_{v}^{i k}= \begin{cases}F^{i k}{ }_{v} & a>4(1-v)^{2} \\ F^{i k}{ }_{v} \mathcal{X}_{v}(a) & 4(1-v)^{2} \geq a \geq(1-v)^{2} \\ 0 & (1-v)^{2}>a,\end{cases}
$$

which lies in $\mathcal{G}(\Omega)$ and equals $F^{i k}{ }_{v}$ outside a cylinder of radius $2(1-v)$ with axis $x=v t$ in the $\rho=0$ plane, whereas it is smoothed down to zero inside a cylinder with half this radius. Note however that $\tilde{F}^{i k}{ }_{v}$ is smooth even on $\mathbb{R}^{4}$. Now $\tilde{F}^{i k}{ }_{v}$ is the best possible approximation for $F^{i k}{ }_{v}$ in $\mathcal{G}(\Omega)$ in the following precise sense: Given any compact $K \subset \Omega$, finally (i.e., $v$ large enough) $\tilde{F}^{i k}{ }_{v}$ equals $F^{i k}{ }_{v}$ on an open neighborhood of $K$.

Putting $v(\epsilon)=1-\epsilon$ we get the desired "embedding" of the field tensor into the Colombeau algebra

$$
\left(F_{v}^{i k}\right)_{v} \mapsto\left(\tilde{F}_{v(\epsilon)}^{i k}\right)_{\epsilon}+\mathcal{N}(\Omega) .
$$

Now we are able both to compute the energy momentum tensor in $\mathcal{G}(\Omega)$, and to make use of the concept of association, which corresponds physically to taking of the ultrarelativistic limit. First we calculate the association relations for the field tensor.

\section{Proposition 1}

$$
\tilde{F}^{i k} \approx\left(\begin{array}{cccc}
0 & 0 & 0 & 0 \\
0 & 0 & -y & -z \\
0 & y & 0 & 0 \\
0 & z & 0 & 0
\end{array}\right) \frac{4 e}{\rho^{2}} \delta(u)
$$

Proof: We have to compute the following limits

$$
\lim _{\epsilon \rightarrow 0} \int_{\Omega} \tilde{F}_{v(\epsilon)}^{i k}(t, x, y, z) \varphi(t, x, y, z) d t d x d y d z
$$

where $\varphi$ is an arbitrary test function in $\mathcal{D}(\Omega)$. We only carry out the calculations for $\tilde{F}^{01}$ and $\tilde{F}^{21}$ as the other components are either related to these by symmetry or the limits can be taken in a closely analogous way. We choose $v$ so large that on an open neighborhood of the support of $\varphi$ we have $\tilde{F}_{v}^{i k}=F^{i k}{ }_{v}$, and for simplicity we set [ $]:=(x-v t)^{2}+\left(1-v^{2}\right) \rho^{2}$. Then we have

$$
\begin{aligned}
\left|\int_{\operatorname{supp}(\varphi)} \tilde{F}_{v(\epsilon)}^{01} \varphi\right| & \leq 2 e\|\varphi\|_{\infty} \iiint\left[\int_{-\infty}^{\infty}\left|\frac{\left(1-v^{2}\right)(x-v t)}{[]^{3 / 2}}\right| d x\right] d t d y d z \\
& =8 \pi e \sqrt{1-v^{2}}\|\varphi\|_{\infty} \iint \frac{1}{\rho} \rho d \rho d t
\end{aligned}
$$

which vanishes in the limit, since the remaining integral has to be taken over a compact set only.

To prove $\tilde{F}_{v(\epsilon)}^{21} \approx\left(4 e y / \rho^{2}\right) \delta(u)$ we first note that for $\tilde{f}_{v(\epsilon)}$, defined from $f_{v}=$ $e y(1+v)(x-v t) /\left(\rho^{2}[]^{1 / 2}\right)$ in the same way as $\tilde{F}_{v(\epsilon)}^{i k}$ is defined from $F_{v}^{i k}$ we have

$$
\lim _{\epsilon \rightarrow 0} \int_{\operatorname{supp}(\varphi)} \tilde{f}_{v(\epsilon)} \varphi=\left\langle\frac{2 e y}{\rho^{2}}[\theta(x-t)-\theta(t-x)], \varphi\right\rangle
$$


where we have used Lebesgue's dominated convergence. Differentiating this relation with respect to $x$ we get the desired result.

Next we compute the energy stress tensor in $\mathcal{G}(\Omega)$ by componentwise multiplication to get

$$
\begin{aligned}
\tilde{T}_{i k v(\epsilon)}= & \frac{e^{2}\left(1-v^{2}\right)^{2}}{2\left((x-v t)^{2}+\left(1-v^{2}\right) \rho^{2}\right)^{3}} \\
& \times\left(\begin{array}{cccc}
\frac{1}{2}(1+v)^{2} \rho^{2} & \frac{1}{2}(x-v t)^{2} & (1+v)(x-v t) y & (1+v)(x-v t) z \\
\frac{1}{2}(x-v t)^{2} & \frac{1}{2}(1-v)^{2} \rho^{2} & -(1-v)(x-v t) y & -(1-v)(x-v t) z \\
(1+v)(x-v t) y & -(1-v)(x-v t) y & (x-v t)^{2}+ & -2\left(1-v^{2}\right) y z \\
& +\left(1-v^{2}\right)\left(z^{2}-y^{2}\right) & \\
(1+v)(x-v t) z & -(1-v)(x-v t) z & -2\left(1-v^{2}\right) y z & (x-v t)^{2}+ \\
& & & +\left(1-v^{2}\right)\left(y^{2}-z^{2}\right)
\end{array}\right)
\end{aligned}
$$

for $a>4(1-v)^{2}$ and smoothed down to zero "inside" in the same way as in the case of $\tilde{F}_{v(\epsilon)}^{i k}$.

\section{Proposition 2}

$$
\begin{aligned}
& \tilde{T}_{i k} \approx 0 \quad \forall(i, k) \neq(0,0) \\
& \tilde{T}_{00} \quad \text { has no associated distribution. }
\end{aligned}
$$

Proof: The calculation for all the components is closely analogous to the one given above with the exception of $T_{00}$, so we shall only examine this one in detail. As a test function $\varphi$ we take $1 / \rho^{2}$ on the set $x, t \in[-N, N], \phi \in[0,2 \pi]$ and $\rho \in\left[r_{0}, r_{1}\right]$ $\left(0<r_{0}\right)$, where $\phi$ is the polar angle in the $(y, z)$-plane, and let it smoothly approach zero "outside". Thus we have for large enough $v$

$$
\begin{aligned}
\int_{\operatorname{supp}(\varphi)} \tilde{T}_{00 v(\epsilon)} \varphi \geq & \frac{e^{2}(1+v)^{2}\left(1-v^{2}\right)^{2}}{4} \int_{-N}^{N} \int_{-N}^{N} \int_{0}^{2 \pi} \int_{r_{0}}^{r_{1}} \frac{\rho^{2}}{[]^{3}} \frac{1}{\rho^{2}} \rho d \rho d \phi d x d t \\
= & \left.\frac{e^{2} \pi(1+v)^{2}(x-v t)}{16 v \rho^{3} \sqrt{1-v^{2}}} \arctan \frac{x-v t}{\sqrt{1-v^{2}} \rho}\right|_{r_{0},-N,-N} ^{r_{1}, N, N} \\
& +\left.\frac{e^{2} \pi(1+v)^{2}}{32 v \rho^{2}} \ln ([\quad])\right|_{r_{0},-N,-N} ^{r_{1}, N, N} \\
& -\left.\frac{e^{2} \pi(1+v)^{2}}{32 v \rho^{2}} \ln \left(1+\frac{(x-v t)^{2}}{\left(1-v^{2}\right) \rho^{2}}\right)\right|_{r_{0},-N,-N} ^{r_{1}, N, N} .
\end{aligned}
$$

In the limit the second term diverges whereas the other two terms vanish.

We see that computing the energy momentum tensor we fall out of the class of Colombeau functions with associated distributions. Taking the square of the two functions $\left(F_{v(\epsilon)}^{12}\right)_{\epsilon}$ and $\left(F_{v(\epsilon)}^{13}\right)_{\epsilon}$, both of them associated to the $\delta$ distribution, we get the generalized function $\left(T_{00 v(\epsilon)}\right)_{\epsilon}=-(1 / 4)\left(\left(F_{v(\epsilon)}^{12}\right)^{2}+\left(F_{v(\epsilon)}^{13}\right)^{2}\right)_{\epsilon}$ not allowing any associated distribution, showing that $\left(T_{00} v(\epsilon)\right)_{\epsilon}$ is an object only defined in the Colombeau algebra. Due to the properties of Colombeau's theory one could subject it to a wide class of nonlinear operations and even use it as a source term for nonlinear PDEs and then check if the resulting generalized function has an associated distribution. 
Of course this result mirrors the fact that if we had computed the energy tensor "naively" from the ultrarelativistic limit of the field tensor in $\mathcal{D}^{\prime}$ we would have obtained $T_{00}=\left(4 e^{2} / \rho\right)$ " $\delta^{2}(u)$," all other components vanishing. This suggests a "commutativity" of the limiting procedure and "multiplication," i. e., the computation of the stress tensor from the field tensor. However, we again point out that no meaningful product can be defined within the framework of distribution theory but only in the Colombeau algebra. As a limiting procedure we have used the concept of association in $\mathcal{G}(\Omega)$, which is not compatible with the product, and so the above-mentioned "commutativity" does not hold in general, as we are going to see in the next section.

\section{THE ULTRARELATIVISTIC LIMIT OF THE REISSNER-NORDSTRØM SOLUTION}

The RN metric representing the gravitational field of a point particle with mass $m$ and charge $e$ in isotropic coordinates is given by

$$
d s^{2}=\left(1-\frac{2 m}{r(\bar{r})}+\frac{e^{2}}{r^{2}(\bar{r})}\right) d t^{2}-\left(1+\frac{m}{\bar{r}}+\frac{m^{2}-e^{2}}{4 \bar{r}^{2}}\right)^{2}\left(d \bar{r}^{2}+d \Omega^{2}\right),
$$

where $r=\bar{r}\left[1+m / \bar{r}+\left(m^{2}-e^{2}\right) /\left(4 \bar{r}^{2}\right)\right]$ is the radial coordinate in a Schwarzschildlike coordinate system. We apply a boost in $x$ direction relative to an asymptotic, Cartesian coordinate system associated with the isotropic radius $\bar{r}$. To obtain a distributionally well defined result in the ultrarelativistic limit we have to rescale the mass and the charge in the following manner:

$$
m=p / \gamma, \quad e^{2}=p_{e}^{2} / \gamma,
$$

where in the limit we keep the momenta $p$ and $p_{e}$ fixed. The rescaling of the mass is well motivated and saves the energy of the particle from diverging due to its finite rest mass by keeping the total energy $p$ fixed and letting $m$ approach zero in the ultrarelativistic limit. The rescaling of the charge is not too well motivated. However, the limit of the metric is given by

$$
d s^{2}=\left\{8 p \ln \rho+\frac{3}{2} \pi \frac{p_{e}^{2}}{\rho}\right\} \delta(u) d u^{2}-d u d w-d y^{2}-d z^{2},
$$

where $u$ and $w$ again denote null coordinates defined as in the last Section. This metric belongs to the class of pp waves and is flat everywhere except on the null plane $u=0$ which is normal to the boost direction and contains the particle.

However, the rescaling of the charge dramatically acts on the limit of the electromagentical field and its stress energy tensor. In a frame associated with null coordinates the boosted field tensor takes the form

$$
F_{i k}=\frac{e\left(1-v^{2}\right) G(\bar{r})}{\left((x-v t)^{2}+\left(1-v^{2}\right) \rho^{2}\right)^{3 / 2}}\left(\begin{array}{cccc}
0 & x-v t & y & z \\
-x+v t & 0 & -v y & -v z \\
-y & v y & 0 & 0 \\
-z & v z & 0 & 0
\end{array}\right),
$$

where the factor $G(\bar{r})=4 \bar{r}^{2}\left(4 \bar{r}^{2}-m^{2}+e^{2}\right) /\left(4 \bar{r}^{2}+4 \bar{r} m+m^{2}-e^{2}\right)^{2}$ arises from the transformation to isotropic coordinates and we again have neglected the bars over $x$ and $t$. Now we embed $\left(F_{i k v}\right)_{v}$ into the Colombeau algebra in the same manner as in the Coulomb case, again denoting the $\mathcal{G}(\Omega)$ function best approximating $F_{i k}$ by $\left(\tilde{F}_{i k v(\epsilon)}\right)_{\epsilon}$. By calculations very similar to those carried out in Sec. II and taking into account the rescaling of mass and charge we get the following association relations. 


\section{Proposition 3}

$$
\tilde{F}_{i k} \approx 0 \quad \forall i, k
$$

Next we compute the stress energy tensor. For $a>4(1-v)^{2}$ we have

$$
\begin{aligned}
& \tilde{T}_{i k}=\frac{p_{e}^{2} G^{2}(\bar{r})}{\left((x-v t)^{2}+\left(1-v^{2}\right) \rho^{2}\right)^{3}} \\
& \left(\begin{array}{cc}
(1-v)^{3 / 2}(1+v)^{7 / 2}\left(g^{11}-g^{00}\right)(x-v t)^{2} / 4 & \left(1-v^{2}\right)^{5 / 2}\left(g^{00}+g^{11}\right)(x-v t)^{2} / 4 \\
+(1-v)^{5 / 2}(1+v)^{9 / 2}\left(g^{00}+g^{11}\right) \rho^{2} / 4 & +\left(1-v^{2}\right)^{7 / 2}\left(g^{11}-g^{00}\right) \rho^{2} / 4 \\
\left(1-v^{2}\right)^{5 / 2}\left(g^{00}+g^{11}\right)(x-v t)^{2} / 4 & (1-v)^{7 / 2}(1+v)^{3 / 2}\left(g^{11}-g^{00}\right)(x-v t)^{2} / 4 \\
+\left(1-v^{2}\right)^{7 / 2}\left(g^{11}-g^{00}\right) \rho^{2} / 4 & +(1-v)^{9 / 2}(1+v)^{5 / 2}\left(g^{00}+g^{11}\right) \rho^{2} / 4 \\
(1-v)^{5 / 2}(1+v)^{7 / 2} g^{00}(x-v t) y & -(1-v)^{7 / 2}(1+v)^{5 / 2} g^{00}(x-v t) y \\
(1-v)^{5 / 2}(1+v)^{7 / 2} g^{00}(x-v t) z & -(1-v)^{7 / 2}(1+v)^{5 / 2} g^{00}(x-v t) z
\end{array}\right. \\
& \left.\begin{array}{cc}
(1-v)^{5 / 2}(1+v)^{7 / 2} g^{00}(x-v t) y & (1-v)^{5 / 2}(1+v)^{7 / 2} g^{00}(x-v t) z \\
-(1-v)^{7 / 2}(1+v)^{5 / 2} g^{00}(x-v t) y & -(1-v)^{7 / 2}(1+v)^{5 / 2} g^{00}(x-v t) z \\
\left(1-v^{2}\right)^{5 / 2} g^{00}\left[(x-v t)^{2}+\left(1-v^{2}\right)\left(z^{2}-y^{2}\right)\right] & -2\left(1-v^{2}\right)^{7 / 2} g^{00} y z \\
-2\left(1-v^{2}\right)^{7 / 2} g^{00} y z & \left(1-v^{2}\right)^{5 / 2} g^{00}\left[(x-v t)^{2}+\left(1-v^{2}\right)\left(y^{2}-z^{2}\right)\right]
\end{array}\right),
\end{aligned}
$$

where

$$
g^{00}(\bar{r})=\left(1+\frac{m}{\bar{r}}+\frac{m^{2}-e^{2}}{4 \bar{r}^{2}}\right)^{2}\left(1+\frac{e^{2}-m^{2}}{4 \bar{r}^{2}}\right)^{-2}
$$

and

$$
g^{11}(\bar{r})=\left(1+\frac{m}{\bar{r}}+\frac{m^{2}-e^{2}}{4 \bar{r}^{2}}\right)^{-2}
$$

and $\tilde{T}_{i k}$ smoothed down to zero "inside" again in the same way. By a rather lengthy calculation, expanding $G(\bar{r}), g^{00}$ and $g^{11}$ and again taking into account the rescaling we get the following.

\section{Proposition 4}

$$
\begin{aligned}
& T_{i k} \approx 0, \quad \forall(i, k) \neq(0,0), \\
& T_{00} \approx \frac{3 p_{e}^{2}}{16 \rho^{3}} \delta(u) .
\end{aligned}
$$

\section{CONCLUSION}

The fact that the "square" $T_{00}$ of the generalized functions $\left(F_{i k}\right)_{\epsilon}$ associated to 0 is not associated to 0 but to the $\delta$ distribution is not surprising from the viewpoint of Colombeau's theory. Association is not compatible with multiplication in the algebra of generalized functions. Thus we can say that the physically unsatisfactory 
situation of a vanishing field but nonzero stress tensor is mathematically perfectly defined. We see the incompatibility of linear idealisations like the $\delta$ distribution and nonlinear computations. Colombeau's theory clearly cannot solve this principal defect but provides us with a language and framework that makes it possible to discuss such situations.

The question of the physical relevance of a zero field producing a nonvanishing energy density clearly has to remain open. But from the mathematical viewpoint such a situation cannot be avoided if one is dealing with distributionally shaped fields.

\section{ACKNOWLEDGMENTS}

Work supported by Doktorandenstipendium der Österreichischen Akademie der Wissenschaften, \# 338.

This work is part of my thesist thank for his extensive help. Also I want to thank M. Grosser, G. Hörmann and M. Kunzinger for many helpful discussions and M. Oberguggenberger for the critical reading of the manuscript.

${ }^{1}$ H. Balasin and H. Nachbagauer, "The ultrarelativistic Kerr-geometry and its energymomentum tensor," Class. Quant. Grav. 12 (3), 707-713 (1995).

${ }^{2}$ H. Balasin and H. Nachbagauer, "Boosting the Kerr-geometry into an arbitratry direction," Class. Quant. Grav. (to be published).

${ }^{3}$ T. Dray and G. 't Hooft, "The gravitational shockwave of a massless particle," Nucl. Phys. B 232, 173-188 (1985).

${ }^{4}$ C. O. Loustó and N. Sánchez, "The ultrarelativistic limit of the Kerr-Newman geometry and particle scattering at the Planck scale," Phys. Lett. B 232, 462-466 (1989).

${ }^{5}$ C. O. Loustó and N. Sánchez, "The curved shock wave space-time of ultrarelativistic charged particles and their scattering," Int. J. Mod. Phys. A 5, 915-938 (1990).

${ }^{6}$ C. O. Loustó and N. Sánchez, "scattering process at the Planck scale," in Second Paris Cosmology Colloquium, edited by H.J. de Vega and N. Sánchez (World Scientific, Singapore, 1995).

${ }^{7}$ P. C. Aichelburg and R. Sexl, "On the gravitational field of a massless particle," J. Gen. Rel. Grav. 2, 303-312 (1971).

8 J.F. Colombeau, "New Generalized Functions and Multiplication of Distributions," (North Holland, Amsterdam, 1984).

9 J. F. Colombeau, "Elementary Introduction to New Generalized Functions," (North Holland, Amsterdam, 1985).

${ }^{10}$ J. F. Colombeau, "Multiplication of Distributions," (Springer, Berlin, 1992).

${ }^{11}$ M. Oberguggenberger, "Multiplication of Distributions and Applications to Partial Differential Equations," (Longman Scientific and Technical, New York, 1992).

12 E. E. Rosinger, "Generalized Solutions of Nonlinear Partial Differential Equations," (North Holland, Amsterdam, 1987).

${ }^{13}$ G. Hörmann and M. Kunzinger, "Nonlinearity and self-interaction in physical field theories with singularities," Integr. Transforms and Special Functions (to be published).

${ }^{14}$ C. J. S. Clarke, J. A. Vickers and J. P. Wilson, "Generalized functions and distributional curvature of cosmic strings," preprint gr-qc/9605060.

${ }^{15}$ R. Steinbauer, "Ultrarelativistischer Limes und Colombeau Theorie," Masters thesis, University of Vienna, 1995. 